\title{
Offline Expansion of XACML Policies Based on P3P Metadata
}

\author{
Claudio Ardagna, Ernesto Damiani, Sabrina De Capitani di Vimercati, \\ Cristiano Fugazza, and Pierangela Samarati \\ Dipartimento di Tecnologie dell'Informazione \\ Università degli Studi di Milano \\ v. Bramante 65, 26013 Crema, Italy \\ \{ardagna, damiani, decapita, fugazza, samarati\}@dti.unimi.it
}

\begin{abstract}
In the last few years XML-based access control languages like XACML have been increasingly used for specifying complex policies regulating access to network resources. Today, growing interest in semantic-Web style metadata for describing resources and users is stimulating research on how to express access control policies based on advanced descriptions rather than on single attributes.

In this paper, we discuss how standard XACML policies can handle ontology-based resource and subject descriptions based on the standard P3P base data schema. We show that XACML conditions can be transparently expanded according to ontology-based models representing semantics. Our expansion technique greatly reduces the need for online reasoning and decreases the system administrator's effort for producing consistent rules when users' descriptions comprise multiple credentials with redundant attributes.
\end{abstract}

\section{Introduction}

Semantic-Web style ontologies are aimed at providing a common framework that allows data to be shared and reused by applications across enterprise and community boundaries. While interest for ontology-based data representation is now growing in many application fields, access control techniques still do not take full advantage of semantic Web metadata. Recent proposals for specifying and exchanging access control policies adopt XML-based languages such as the eXtensible Access Control Markup Language (XACML) [4]. Although very expressive and flexible, XACML is severely limited by the comparatively low expressive power of formalisms used to describe resources and users requesting them.

In this paper we present a practical and efficient approach for increasing XACML expressive power by incorporating into XACML policies ontologybased resource and subject descriptions based on the standard P3P base data schema [8]. In particular, we describe how XACML conditions can be expanded taking into account ontology-based models representing user profiles and resources semantics. Our expansion technique greatly reduces the need for on-line 


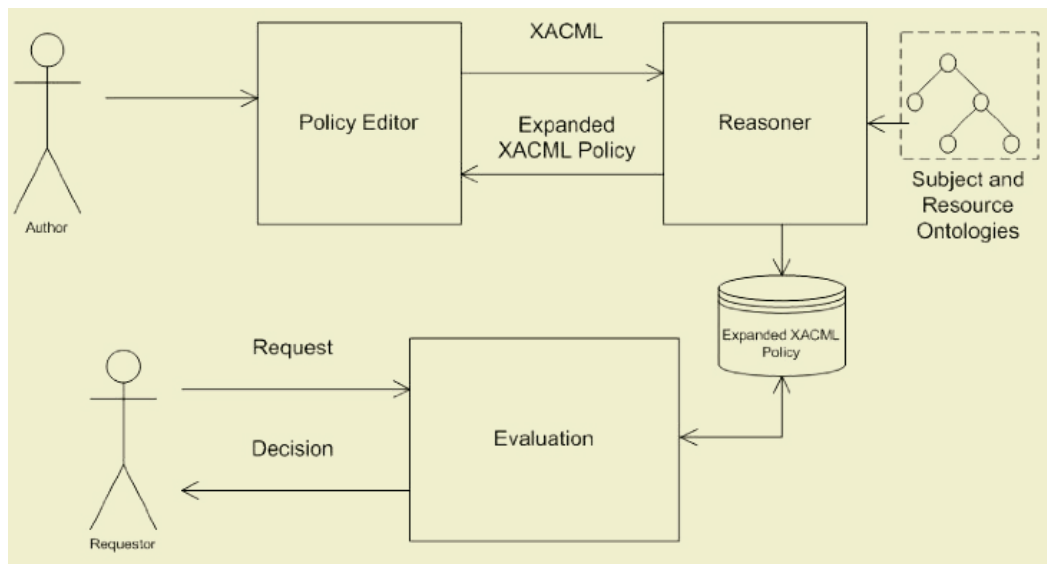

Fig. 1. Scenario

reasoning, relieving the (potentially heavy) computational burden of supporting resource and users' semantics in policy definition and evaluation. As far as user descriptions are concerned, we rely on P3P-based credentials [8], which are increasingly used to represent subject-related personal information in privacy policies. In our approach, the standard P3P base data schema for credential definition is converted into semantic-Web style metadata that can be easily checked in the framework of policy evaluation ${ }^{1}$. Figure 1 illustrates the scenario we consider. The XACML policies are created via the Policy editor component. The Reasoner takes such XACML policies together with the subject and resource ontologies as input and computes the expanded XACML policies including semantically equivalent additional conditions. These conditions, specified in disjunction with the original ones, allow for increasing the original policy's expressive power. Our semantically expanded XACML policies can be straightforwardly used as a replacement of the original ones or, more interestingly, can be evaluated side by side with them, flagging cases of inconsistency in the policies' semantics.

Therefore, our solution allows the definition of rules based on generic assertions defined over concepts in the ontologies that control metadata content. The result is a semantic-aware policy language exploiting the high expressive power of ontology-based models. The remainder of this paper is organized as follows. Section 2 describes our semantic-Web style representation of the standard P3P base data schema. Section 3 shows a simple example of XACML policy expansion, deriving possible alternatives obtained by straightforward reasoning over context information. Section 4 illustrates how the reasoning process derives the actual credential users should provide to be granted access to a given resource by taking into account an explicit representation of resources. Finally, Section 5 shows how a complex condition is modeled and how the ontology is used to deduce some semantically equivalent alternatives.

\footnotetext{
${ }^{1}$ More specifically, the ontology used in this paper relies on the revised P3P base data schema introduced in our previous work $[3,6]$
} 


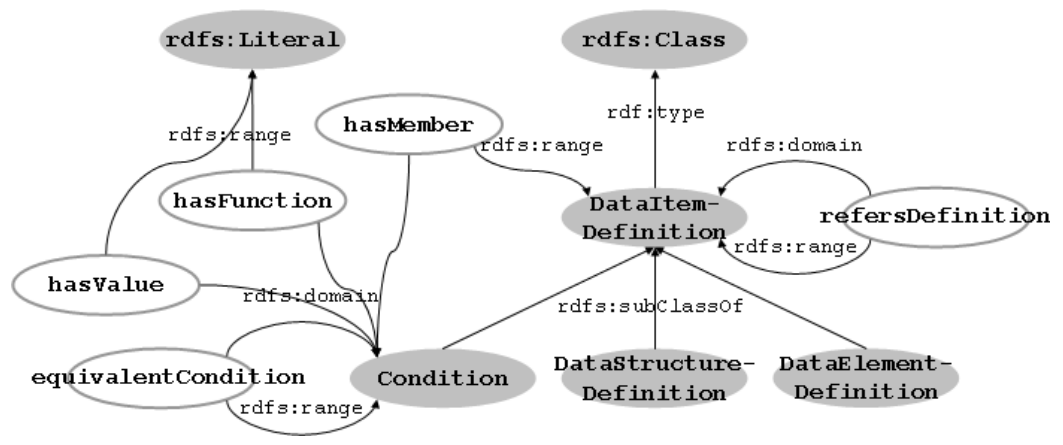

Fig. 2. The language layer defining the building blocks of our RDFS-based representation of the standard P3P base data schema

\section{Representing Heterogeneous Credential Information}

We start from a simple RDFS representation of the standard P3P base data schema. Fig. 2 shows the first level of RDF Schema definitions [10] (the language layer) for our semantic-Web style representation of the standard P3P base data schema [8]. Here, classes have a grey background color while properties have borders and no background color. Root class DataItemDefinition is sub-classed by DataStructureDefinition and DataElementDefinition to model P3P data structures and data elements, respectively, and by a class Condition used to model policy conditions by means of the associated properties hasFunction, hasMember, and hasValue. More precisely, these properties represent the evaluation function, the variables, and the literal values used in the condition, respectively. Conditions can feature alternatives by means of property equivalentCondition. We denote the inclusion between DataItems with property refersDefinition whose domain and range (in RDFS terms rdfs: domain and rdfs:range) coincide with the DataItemDefinition class. This property, together with the $\mathrm{rdf} \mathrm{s}$ : subClass Of property, will be used throughout the paper to query the knowledge base for alternatives to data items.

Below the language layer, the ontology layer (see Fig. 3) comprises data elements and structures represented as classes and linked with each other by means of the refersDefinition property. This layer models the semi-lattice structure of the P3P base data schema [6]. At the bottom of the model, the instance layer contains the actual negotiable user credential expressed as instances of the classes defined by the ontology layer. Credentials are connected by the refersInstance property, modeling the tree structure linking literal values to nodes representing credential instances as a whole. For the sake of simplicity, rather than bending the built-in inference rules associated with ontology languages like OWL [7], we rely on plain RDFS and define from scratch a rule set representing the reasoning patterns required by our application. In the following, the examples make use only of the implications in the ontology layer, hence the instance layer is not further described. 


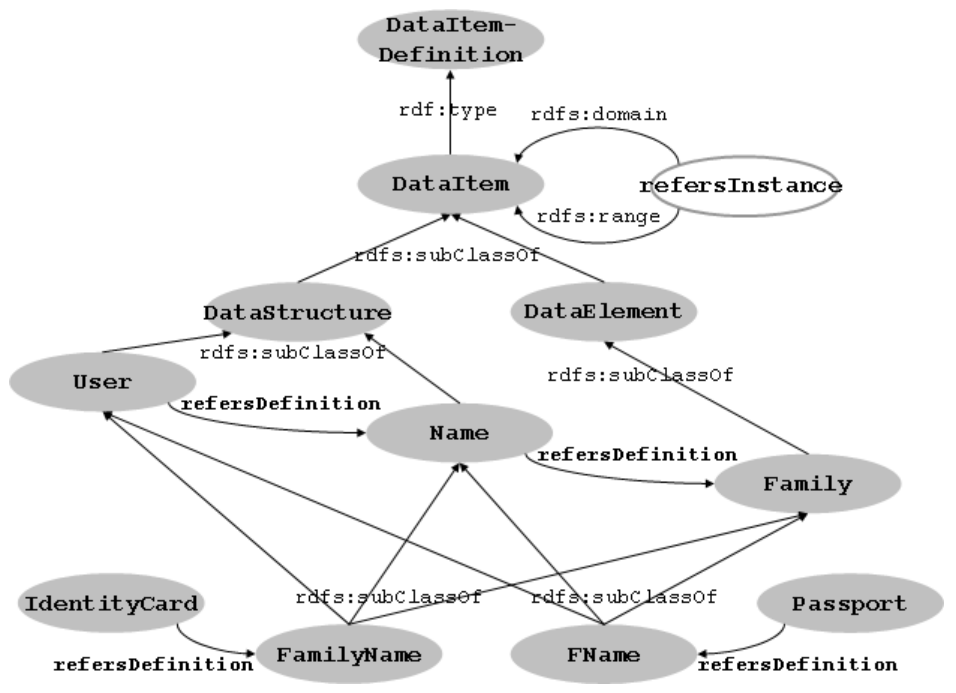

Fig. 3. The ontology layer integrating the standard P3P base data schema with credential definitions

\section{Referencing Credential Information in Policies}

We are now ready to describe some worked out examples of ontology-based policy expansion. In the first example, we show how using an ontology to link independent credentials to the P3P base data schema allows for easily specifying alternatives. Fig. 4 shows an XACML condition that references data items of the extended context provided by the ontology via the urn: ...:BaseDataSchema:User:Name:Family URN, matching it with the literal "Rossi". From the URN we can note that the referenced data items:

- belong to the underlying P3P base data schema and can correspond to different alternatives (one for each possible credential);

- have type Family, which is part of the Name context associated with the User requiring a resource or service.

From this URN, the following RDQL [11] query can be derived.

SELECT ?DataElement

WHERE

(<http://../BaseDataSchema\#Family $><$ http://../BaseDataSchema\#refersDefinition $>$ ?DataElement) (?DataElement rdfs:type <http://../BaseDataSchema\#SubjectAttribute $>$ )

The query extracts the referenced data items from the P3P base data schema (note that class SubjectAttribute allows for distinguishing subject credentials from resources types). Let us now examine in more details the (hopefully rather self-explanatory) RDQL syntax. First of all, in the selection clause we collect all ontology nodes referenced by the Family node. Since Family is a leaf and refersDefinition is a reflexive property (i.e., ?Credential 


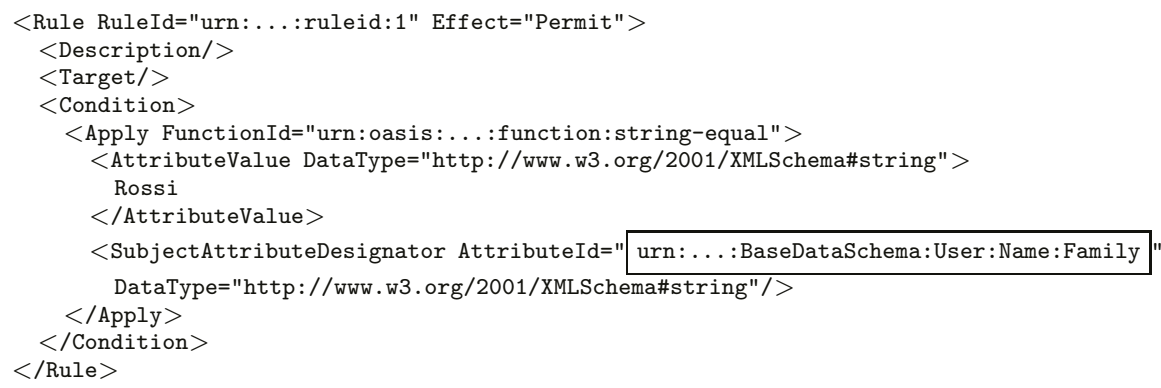

Fig. 4. A simple XACML condition referencing the extended context

refersDefinition ?Credential holds for any candidate credential), the only result is:

$<$ http://.../BaseDataSchema\#Family $>$

Then, we query the information base for data items semantically equivalent to BaseDataSchema\#Family. Note that the other elements specified in the URN (i.e., User and Name) act as constraints. We then derive the following RDQL query:

\section{SELECT ?DataElement}

\section{WHERE}

(?DataElement rdfs:subClassOf <http://.../BaseDataSchema\#User $>$ )

(?DataElement rdfs:subClassOf <http://../BaseDataSchema\#Name $>$ )

(?DataElement rdfs:subClassOf <http://../BaseDataSchema\#Family $>$ )

(?DataElement rdfs:type <http://../BaseDataSchema\#SubjectAttribute $>$ )

The RDFS reasoning engine returns two results:

$<$ http://.../Passport\#FName $>$

$<$ http://.../IdentityCard\# FamilyName $>$

The entities are then mapped to the URNs urn: . . : Passport:FName, and urn: . . : IdentityCard:FamilyName, respectively, and the original condition is expanded as illustrated in Fig. 5.

Note that, as long as the two parties agree on the extended context, our expansion procedure does not affect the asymptotic complexity of evaluation, since the expanded condition can still be evaluated by applying XACML standard functions to literal values. Our policy expansion process has therefore the advantage of hiding the complexity of the structure of the user description metadata.

\section{Referencing Proprietary Representations of Resources}

P3P base data schema is normally used for describing user-related personal information. However, the scope of our technique can be easily enlarged to encompass resource descriptions. The availability of a common data schema allows 


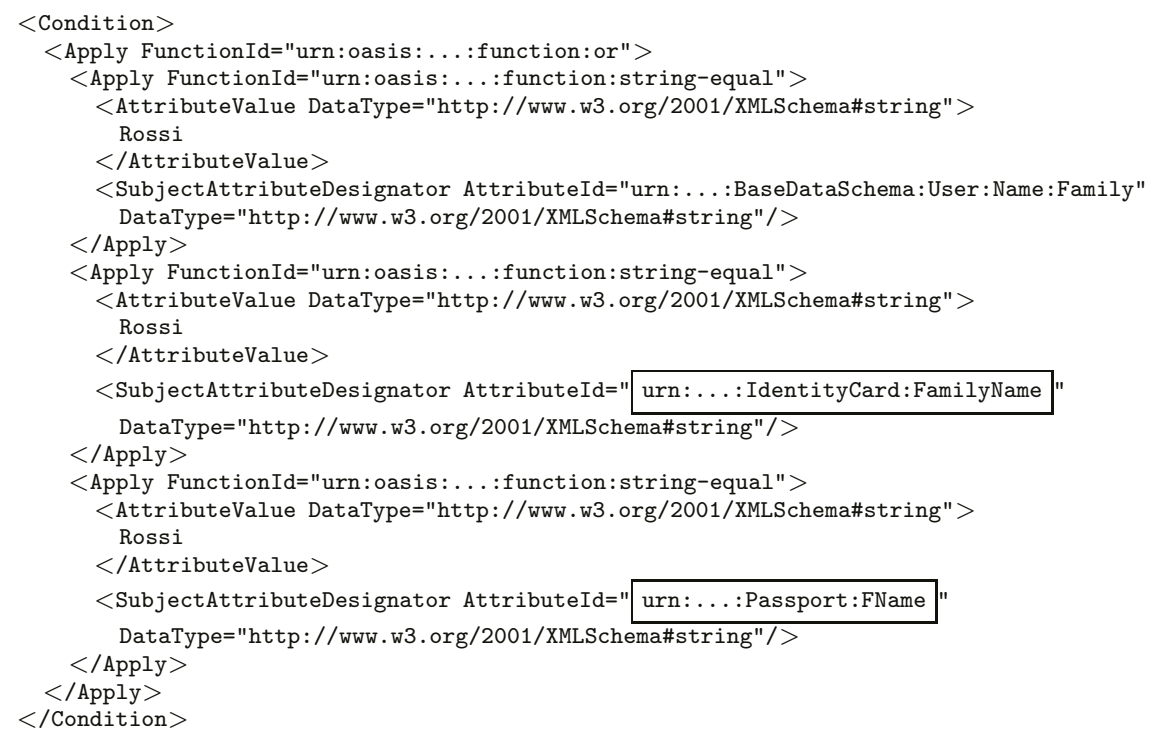

Fig. 5. The XACML condition of Fig. 4 after the expansion

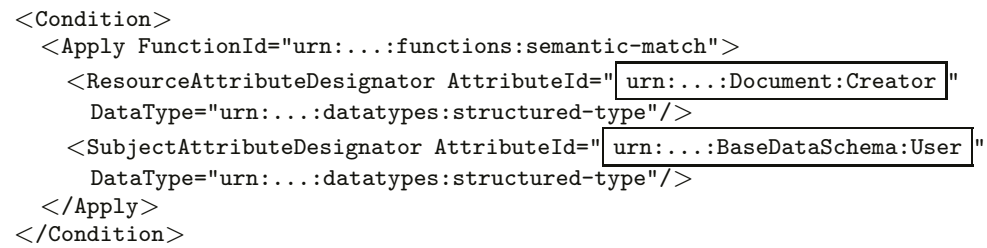

Fig. 6. XACML condition referencing an arbitrary categorization of resources

for rooting an arbitrary representation model for describing resources metadata: actual credentials can then be associated with this information. Following the standard XACML approach, our second example introduces a custom matching function, called semantic-match, that will be applied to structured data items, indicated by the custom data type structured-type. As an example, consider the XACML condition in Fig. 6. This condition is evaluated to true when the Document: Creator data structure describing a resource matches the User data structure from the base data schema. The task of finding the right credential against which to match the resource's Creator can then be left to the reasoning engine.

In this example, our semantic-match function works as follows:

- First, it retrieves data items identifying a document's creator (for the sake of conciseness, we assume that authors are uniquely defined by their first name, last name, and e-mail). Here, class ResourceAttribute allows for distinguishing resource types from subject credentials: 
SELECT ?DataElement

WHERE

(<http://../Documents\#Creator $><$ http://../BaseDataSchema\#refersDefinition $>$ ?DataElement)

(?DataElement rdfs:type <http://../BaseDataSchema\#ResourceAttribute $>$ )

According to our assumption, this query returns three values:

$<$ http://../Document\#FirstName $>$

$<$ http://.../Document\#FamilyName $>$

$<$ http://.../Document\#EmailAddress $>$

- Since resources descriptions are not credentials, their definitions need not be shared. Thus, for each of the data items retrieved, the corresponding superclasses in the base data schema are identified ${ }^{2}$. For instance, the following RDQL query selects data items in the base data schema that are equivalent to data element FirstName.

SELECT ?DataElement

WHERE

(<http://../Documents\#FirstName > rdfs:subClassOf ?DataElement)

(?DataElement rdfs:type <http://../BaseDataSchema\#SubjectAttribute>)

The result of this query is

$<$ http://../BaseDataSchema\#Given $>$

- At this point, all data items can be retrieved as in Section 3, taking into account the User constraint in the SubjectAttributeDesignator:

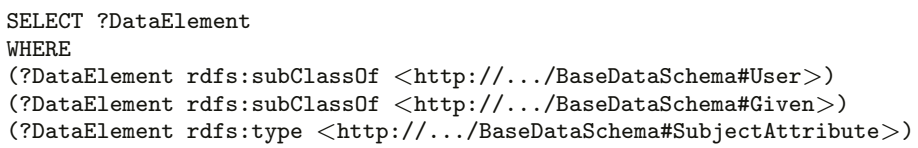

This query returns two values:

<http://../Passport\#GName $>$

$<$ http://.../IdentityCard\#FirstName $>$

After translating all the alternatives into URNs, the condition is expanded according to the translation's results. Once again, we remark that the expanded policy is fully compliant with the XACML standard schema defined in [4]. For the sake of conciseness, the (rather verbose) result of the expansion is shown in Appendix A.

${ }^{2}$ Note that these super-classes are themselves leaves induced in the P3P base data schema by the refersDescription property 


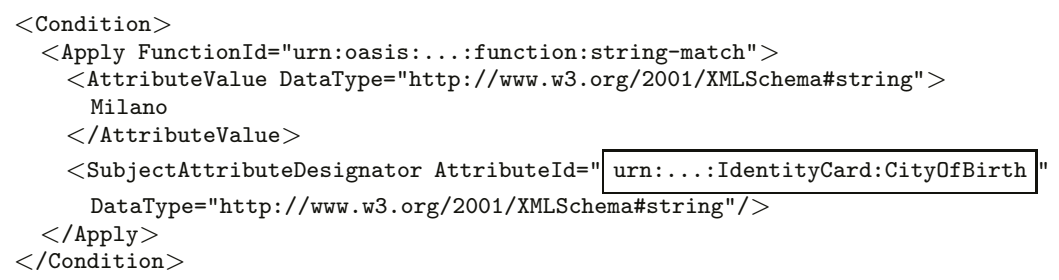

Fig. 7. A condition on the city of birth of the requestor

\section{Expressing Advanced Semantics-Aware Conditions}

In this Section, our expansion technique is extended to take into account not only the metadata context being referenced by a policy, but also how data items are combined or evaluated in conditions. As we will see, complex translations schemes can be defined, leading to equivalent conditions in terms of the attributes being compared, the function being applied, and also the right-end value of the comparison. In other words, our example will take into account not only the context composed of attributes associated with subjects and resources, but also the operational semantics of the policy language describing the rules. Here, metadata represents not only the data items being exchanged, but also the conditions applied to them.

The conditions shown in Figure 7 states that the city of birth of the requestor should be equal to Milano.

In triple format this condition can be expressed as follows:

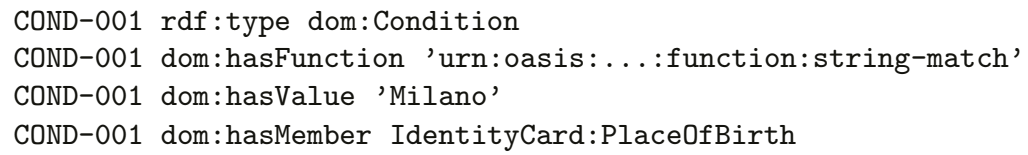

Our expansion technique derives an equivalence between these triples and the ones below:

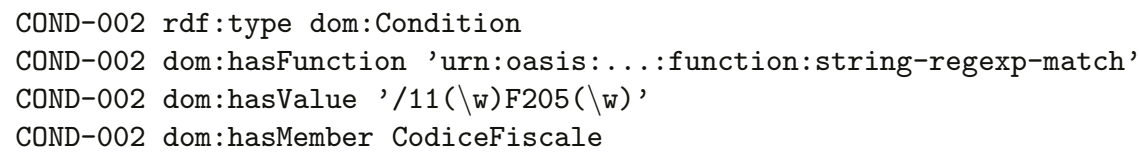

In other words, we can generate the following assertion:

COND-001 dom:equivalentCondition COND-002

Note that condition COND-002 has left and right terms different from those of COND-001 and also uses a different evaluation function. However these two conditions are equivalent because the Italian tax code, called codice fiscale, is a 16 digits alphanumeric code uniquely defined by the first name, last name, gender, date, and city of birth. The city code F205 appearing in positions 12 to 15 , indicates Milano as the city of birth and therefore the original condition 


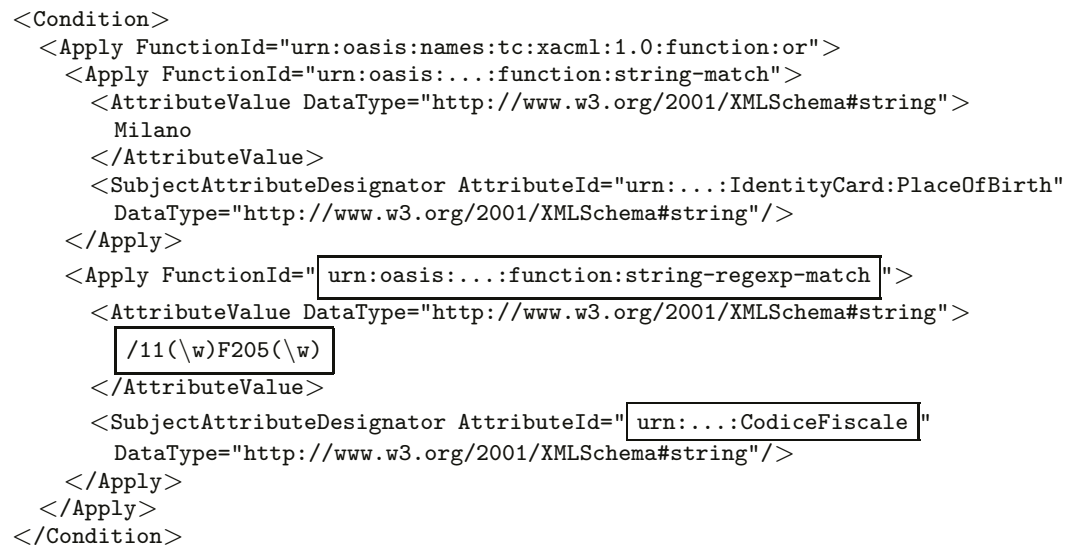

Fig. 8. The condition of Fig. 7 after the expansion

can also be expressed in XACML by matching the codice fiscale against the regular expression '/11( \w)F205(\w)'. The expanded condition is represented in Fig. 8.

Computationally, the equivalence between the two conditions can be checked by direct mapping with tabled values, such as the city codes appearing in the CodiceFiscale, or else provided by means of numeric or string conversion functions.

\section{Extending XACML to Support Complex Conditions}

The previous examples have shown how we can expand XACML conditions expressing a predicate (e.g., equality) between an attribute and a literal or between two attributes by means of an ontology based on the standard P3P data schema. One step further toward increasing the policy language expressive power beyond plain XACML would require dealing with more complex logic conditions, including variables and quantifiers. For instance, the complex condition "User $X$ can see document $Y$ if there exists at least another document $Z$ with the same creation date" cannot be expressed in plain XACML. Also, evaluating this kind of conditions is known to be a difficult computational problem [2]. For this, we need to define a different XML-syntax, based on a BNF grammar like the one shown below:

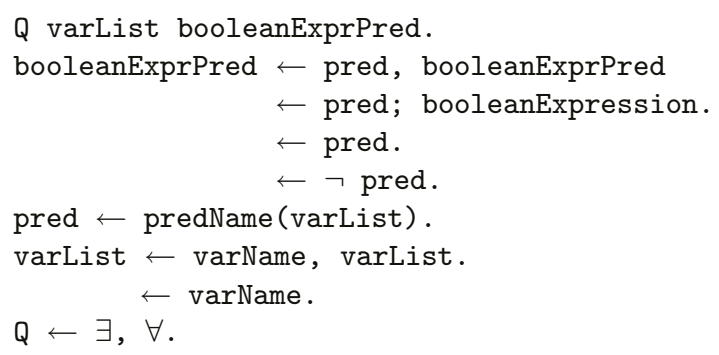


Note that with this approach the evaluation mechanism of the new policy language will need to perform ontology-based reasoning as an integral part of the policy evaluation mechanism rather than using it to explicitly expand policies. The evaluation of complex conditions requires a component (currently being developed [1]) traslating XML-based logic conditions into RDQL queries to be submitted to an ontology-based reasoner during the evaluation phase. Online reasoning about conditions, of course, will require careful design in order to keep the computational burden of policy evaluation under control; also, it may lead to unexpected results, as the effects on policy evaluation of the semantic information stored in the ontology are not available for inspection. For this reason it might be necessary to publish, for transparency, also the inference rules used for the evaluation. We plan to deal with this subject as future work.

\section{Conclusions}

We have illustrated how XACML policies can be expanded on the base of ontology-based resource and subject descriptions encoding the P3P base data schema. Our approach can be used with any P3P data schema to derive, given a specified policy, a policy that includes semantically equivalent additional conditions on users and resource description metadata. The advantage of our solution is twofold. First, it permits to automatically extend available access control policies taking into account resources whose metadata express similarity with other resources already mentioned in the policies. Second, it allows to check consistency of existing policies with respect to different resources and users having a similar semantic status.

\section{Acknowledgments}

This work was supported in part by the European Union within the PRIME Project in the FP6/IST Programme under contract IST-2002-507591 and by the Italian MIUR within the KIWI and MAPS projects.

\section{References}

1. C.A. Ardagna, E. Damiani, S. De Capitani di Vimercati, M. Cremonini, and P. Samarati - Towards Identity Management for E-Services - In Proc. of the TED Conference on e-Government Electronic democracy: The challenge ahead, Bozen, Italy, March 2005.

2. P. A. Bonatti, P. Samarati - A Uniform Framework for Regulating Service Access and Information Release on the Web - Journal of Computer Security 10(3): 241$272(2002)$

3. E. Damiani, S. De Capitani di Vimercati, C. Fugazza, and P. Samarati - Extending Policy Languages to the Semantic Web - In Proc. of ICWE 2004, Munich, 2004, Lecture Notes in Computer Science 3140. 
4. eXtensible Access Control Markup Language (XACML) - Organization for the Advancement of Structured Information Standards - http://www.oasisopen.org/committees/tc_home.php?wg_abbrev=xacml

5. Jeff Z. Pan, Ian Horrocks - Metamodeling Architecture of Web Ontology Languages - In Proc. of the Semantic Web Working Symposium 2001

6. P. Ceravolo, E. Damiani, S. De Capitani di Vimercati, C. Fugazza, and P. Samarati - Advanced Metadata for Privacy-Aware Representation of Credentials - In Proc. of the ICDE Workshop on Privacy Data Management (PDM 05)

7. OWL Web Ontology Language - Overview - W3C Recommendation, December 2003 - http://www.w3.org/TR/owl-features/

8. Platform for Privacy Preferences (P3P) - W3C Recommendation, 16 April 2002 - http://www.w3.org/TR/P3P/

9. Privacy and Identity Management for Europe (PRIME) - European RTD Integrated Project - http://www.prime-project.eu.org/

10. RDF Vocabulary Description Language (RDFS) - W3C Recommendation, 10 February 2004 - http://www.w3.org/TR/rdf-schema/

11. $R D Q L-A$ Query Language for $R D F$ - W3C Member Submission, 9 January 2004 - http://www.w3.org/Submission/2004/SUBM-RDQL-20040109/

\section{A Expansion of the Condition in Fig. 6}

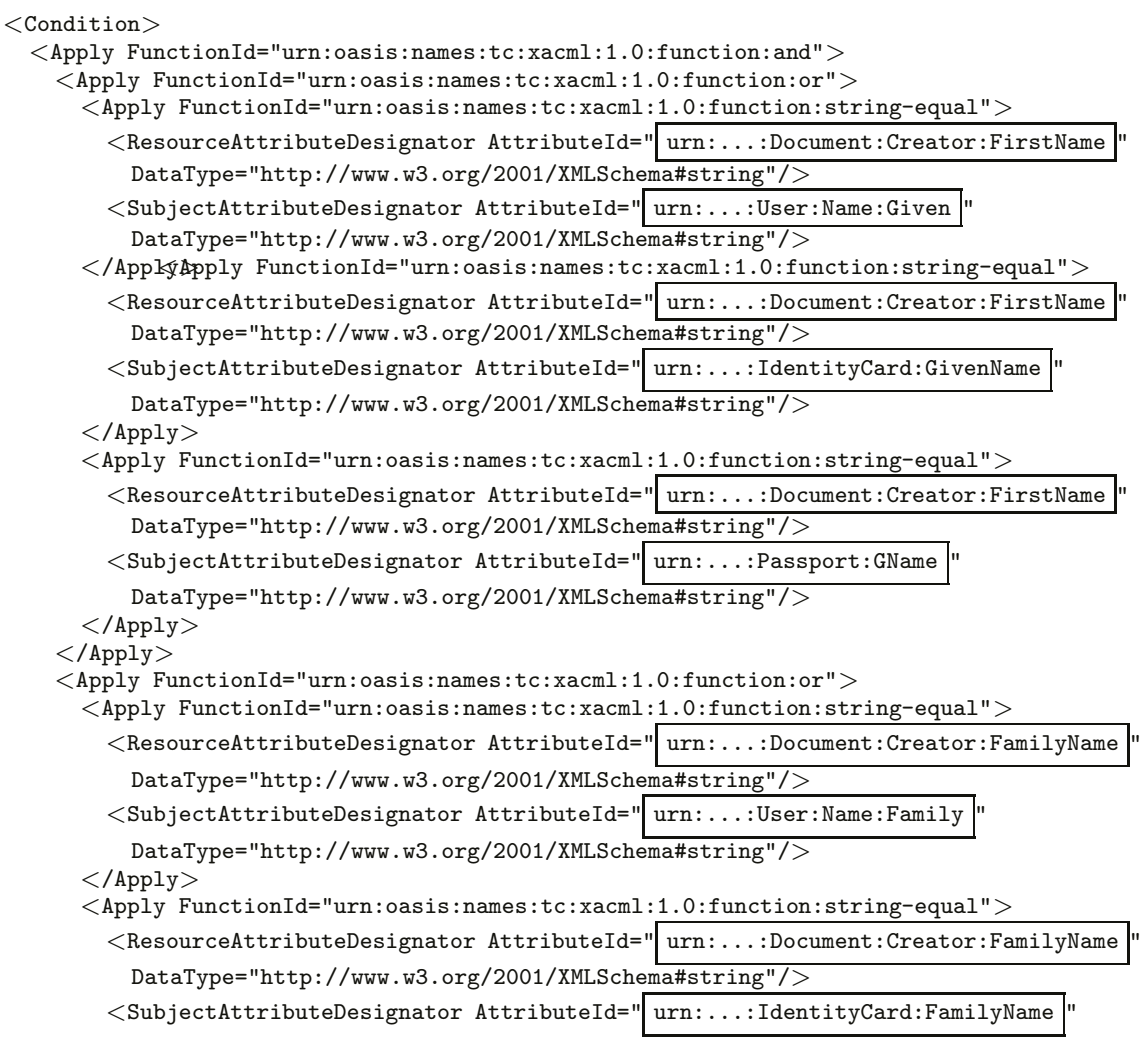




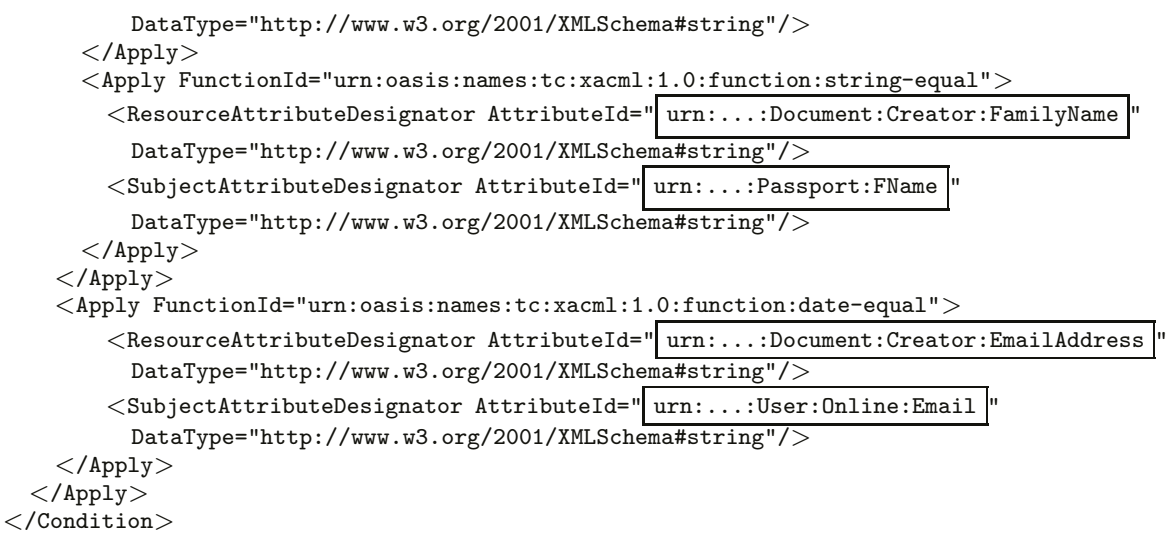

\section{B RDQL Basics}

RDQL queries have the following general form:

\section{SELECT ?a}

FROM <http://input-model.rdf $>$

WHERE (?a, <http://some-predicate $>$, ?b)

AND $? \mathrm{~b}<5$

Question marks indicate variables, each variable in the SELECT clause determines a column in the output. The FROM clause allows for selecting a specific file as the input model; this functionality is not used in the paper.

The WHERE clause simply defines triples that must be found in the knowledge base for a result to be selected: in the example, elements eligible for the ?a placeholder must have property some-predicate linking to some element ?b.

Finally, the AND clause allows for evaluating literal values according to a set of standard functions: in the example, the element ?b linked to a candidate result ?a must evaluate as $<5$. Also this functionality is not used in the paper. 